\title{
Large-Aperture Mirror Array (LAMA) - conceptual design for a distributed-aperture 42-meter telescope
}

\author{
Paul Hickson ${ }^{1 \mathrm{a}}$ and Kenneth M. Lanzetta ${ }^{2 \mathrm{~b}}$ \\ ${ }^{\mathrm{a} U n i v e r s i t y ~ o f ~ B r i t i s h ~ C o l u m b i a ; ~}{ }^{\mathrm{b}}$ Stony Brook University
}

\begin{abstract}
We propose a high-density coherent array of eighteen 10-meter liquid-mirror telescopes. Each telescope is equipped with a reflective corrector system that enables it to point and track within an eight-degree diameter accessible field of view centered on the zenith. Equipped with adaptive optics, each telescope will deliver a diffraction-limited beam to a common focal plane. There, a dichroic optical-infrared camera will provide simultaneous imaging in six wavelength bands from 0.6 to $2.2 \mathrm{um}$. Operating coherently as a Fizeau imaging interferometer, the array would be capable of achieving 4 mas resolution at $1 \mathrm{um}$ and a point source sensitivity of $40 \mathrm{pJy}\left(I_{A B}=34.9\right)$ in a single 30 min exposure. With incoherent beam recombination it would be capable of 25 mas resolution and a point-source detection limit of 250 pJy $\left(I_{A B}=32.9\right)$ in a single $30 \mathrm{~min}$ exposure. The telescope uses established technologies and could be built in a relatively short time.
\end{abstract}

Keywords: telescopes, interferometers, adaptive optics, liquid mirrors

\section{INTRODUCTION}

While most concepts for the next generation of very-large optical/infrared telescopes employ giant segmented primary mirrors, the alternative approach of combining beams from multiple monolithic mirrors has many advantages. As Beckers $^{1}$ points out, multiple-mirror telescopes (MMTs) occupy less volume, are potentially more rigid and less susceptible to wind, are less costly, and require a minimum of new technology.

In this paper we extend the MMT idea further. Unlike the original MMT, the current Large Binocular Telescope (LBT), or the Extremely-Large MMTs discussed by Beckers, the individual primary mirrors in our distributed-aperture concept do not share a common mount. Furthermore, they are fixed both in location and orientation, directed at the zenith. This greatly reduces the cost and complexity of the mechanical structure, and allows the possibility of employing low-cost elements such as rotating liquid mirrors ${ }^{2,3,4}$.

With fixed primary mirrors, pointing and tracking must be accomplished by other optical elements. Recent work ${ }^{5}$ has shown that optical correctors are now possible that allow pointing and tracking within an 8-degree diameter area, giving typical exposure times of order $30 \mathrm{~min}$. We argue that, for many scientific programs of high interest, these limits do not significantly restrict the impact of the telescope.

These considerations have led us to a concept for a low-cost very-large optical telescope that we call the Large-Aperture Mirror Array (LAMA). The baseline design consists of eighteen 10-meter liquid-mirror telescopes deployed in a closepacked hexagonal array. The effective area is that of a 42-meter telescope and the resolution, with coherent operation, is comparable to that of a 54-meter telescope. Located at a low latitude site, the telescope would be able to access $7 \%$ of the entire sky, and achieve point-source detection limits unsurpassed by any telescopes proposed for the next decade.

${ }^{1}$ hickson@physics.ubc.ca; phone 1604822 3853; fax 1604822 5324; http://www.astro.ubc.ca/people/hickson; University of British Columbia, Vancouver, BC V6T1Z1, Canada; ${ }^{2}$ lanzetta@ sbastr.ess.sunysb.edu; phone 1631632 8222; fax 1631632 8176; Stony Brook University, Stony Brook, NY 11794, USA 


\section{DESIGN OVERVIEW}

The LAMA concept consists of a large number of individual afocal telescopes that produce compressed collimated beams, transport systems that convey these beams to a central location, and a beam combiner that directs them to a common focus. In addition to these main components, there are pointing and tracking systems, adaptive optics systems, pathlength compensation and phase-tracking systems.

There is considerable flexibility choosing the sizes of the individual apertures, the number of apertures, and their geometrical configuration. The total light collecting area is determined by the diameter $D$ of each aperture and the number of elements $N$, and the resolution is determined by the overall diameter $d$ of the configuration. An important aspect is the image contrast, which affects the sensitivity of the telescope to point sources. For a dilute configuration, most of the power is diffracted into side lobes of the point spread function (PSF). It can be shown that the fraction of the total power that appears within the central PSF, which has angular size $\theta \approx \lambda / d$, is equal to the filling factor $f \approx N(D / d)^{2}$ of the array ${ }^{6}$. Therefore, while sparse arrays can provide high resolution images, the low fraction of light contained within the image core limits their sensitivity. For this reason, we choose to deploy our apertures in a close-packed configuration in order to provide maximum sensitivity. The highest-density configurations are hexagonal, as illustrated in Fig. 1. In this figure, $D^{*}$ is the diameter of a single telescope having the same light collecting area and $\mathscr{S}=N(f d / D)^{2}$ is a dimensionless measure of the sensitivity of the array, inversely proportional to the time required to reach a given signal-to-noise ratio, compared to that of a single element, for background-limited observations.
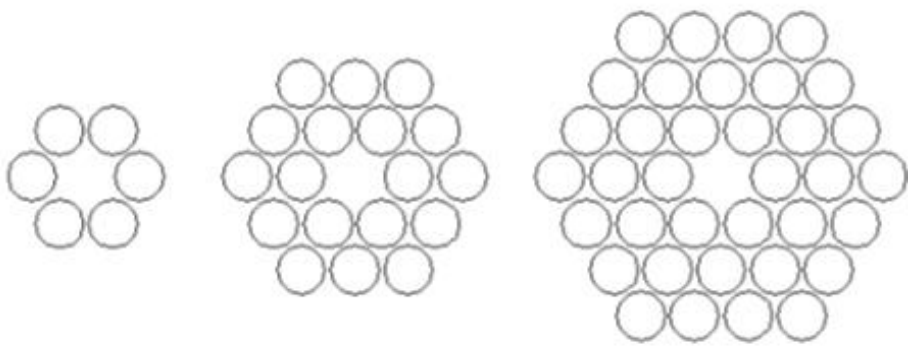

\begin{tabular}{|l|l|l|l|}
\hline$N$ & 6 & 18 & 36 \\
\hline$d / D$ & 3.2 & 5.4 & 7.6 \\
\hline$f$ & 0.64 & 0.71 & 0.73 \\
\hline$D^{*} / D$ & 2.45 & 4.24 & 6.0 \\
\hline $\mathscr{S}$ & 39.3 & 372.7 & 1518 \\
\hline
\end{tabular}

Fig. 1. Three possible high-density array configurations and their characteristic parameters

For the individual apertures, it makes sense to use the largest monolithic mirrors available. For conventional glass mirrors this means a diameter of about 8 meters. For liquid mirrors there are no strict limits on size, but a prudent extension of current technology dictates a size in the range 10-12 meters. This choice results in reasonable sizes for the tracking and relay optical elements and is well-suited to current adaptive-optics technology.

The number of elements is determined primarily by cost, and quantized by the desire to fill the entrance pupil as completely as possible. For the baseline LAMA design, we have adopted the 18-element configuration shown in Fig. 1.

\section{TECHNICAL CONSIDERATIONS}

\subsection{Primary mirrors}

The primary mirrors can be spherical or parabolic. Spherical mirrors are preferable if conventional glass mirror technology is used. This choice simplifies the tracking optics and allows pointing and tracking over a wide angle, limited only by vignetting. However, liquid-mirrors are an order of magnitude less costly and are therefore attractive. Laboratory tests $^{2}$, theoretical analysis ${ }^{7}$, and analysis of images obtained with the 3-meter NASA Orbital Debris Observatory (NODO), all indicate that these mirrors have diffraction-limited surface quality. The largest liquid-mirror telescope is the 6 -meter Large Zenith Telescope (LZT) ${ }^{8}$. The cost of the primary mirrors for these instruments is approximately $\$ 5000$ per square meter, which is much less than that of glass mirrors. 
Current liquid-mirror telescopes employ mercury to form the reflecting surface. Although a decade of experience at NODO, and five other facilities, show that this substance can be safely controlled, possible alternatives such as ionic liquids are being investigated.

The primary mirror technology employed by the LZT can be readily scaled to larger sizes. At present, the main consideration in moving to much larger diameters is the effect of vortices in the air above the mirror, induced by the local linear velocity of the rotating mirror. These vortices are visible in laboratory tests and in defocused star images from the 3-meter NODO mirror, but their effect on the optical surface is relatively minor, and becomes negligible if the thickness of the mercury layer is reduced to less than a millimeter. Nevertheless, it does not seem prudent at this time to go much beyond a factor of two increase in diameter from the LZT. There are other reasons for not wanting the primary mirrors to be too large. The tracking optics design dictates a primary focal ratio of order $f / 2.5$. Increasing the size of the primary mirrors increases the size and cost of the tracking optics, and the size and cost of the telescope enclosure. Therefore the mirrors of the current baseline design have diameters of 10 meters. Experience with the LZT could allow us to increase this to 12 meters, which would effectively make LAMA a 50-meter telescope.

Operational aspects of liquid-mirror telescopes are well documented ${ }^{9}$. More than 20 years of cumulative experience now exist with the operation and maintenance of these telescopes. Liquid mirrors are reliable and easily maintained and cleaned. Health and safety procedures regarding mercury are well understood.

\subsection{Tracking optics}

Although several off-axis corrector designs have been proposed for liquid mirrors ${ }^{10,11}$, these are not suitable for LAMA as they do not correct distortion. The resulting zenith-angle dependent distortion would result in unacceptable image elongation for all but the shortest exposure times. Recently, a completely distortion-free design has been proposed ${ }^{5}$. In its simplest form, a concave secondary mirror located behind the prime focus receives light from a spherical primary mirror and produces an afocal beam that is directed to the beam combiner by a set of flat mirrors. The spherical aberration of this system is removed by an aspheric "Schmidt" corrector located in the afocal beam. Pointing and tracking is achieved by rotating the secondary mirror and corrector about the center of curvature of the primary mirror. The symmetry of the system ensures that no off-axis aberrations are present, other than field curvature which is easily controlled.

With a parabolic primary mirror, the system is more complex. In addition to the Schmidt corrector, an aspheric "parabolic compensator" is placed in the afocal beam at a location conjugate to the primary mirror. This corrector introduces a phase advance that cancels the relative phase delay introduced by the parabolic primary mirror, making the system equivalent to the spherical case. During tracking, this compensator is moved transversely in order to keep its axis conjugate to that of the primary mirror. With the help of adaptive optics, that flattens the wavefront at the center of the field (but does not remove field-dependent aberrations), this system can produce diffraction-limited image quality (Strehl ratio $S>0.7$ ) over a 1 arcmin diameter instantaneous field of view up to four degrees from the zenith.

As Fig 2 shows, the baseline tracking system design requires six mirrors, one of which is flat. Light reflected from the primary mirror enters from below through a hole in M3. The prime focus occurs midway between M2 and M3. These two mirrors form an image of the primary mirror at M4, the parabolic compensator. M7 is the Schmidt corrector, which produces the final afocal beam. M6 is a small flat mirror that rotates as the system tracks in order to keep the output beam horizontal. As the system tracks, the configuration rotates about the center of curvature of the

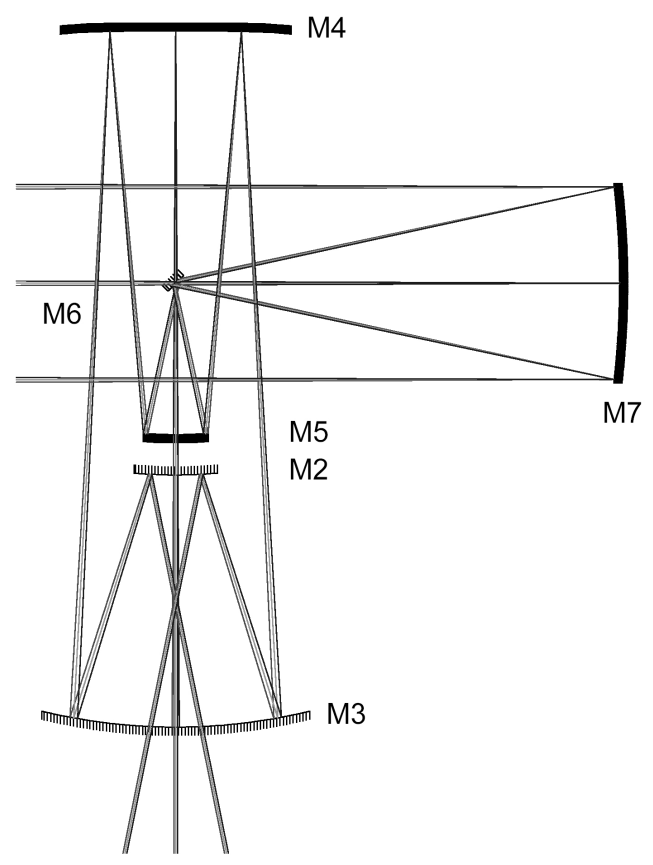

Fig. 2. Optical configuration of the LAMA tracking system. 
primary mirror while M4 also moves transversely.

\subsection{Adaptive optics}

Adaptive optics is an essential part of the LAMA concept. Each 10-meter telescope is equipped with an AO system that removes atmospheric phase errors, and the zero-field geometrical phase errors of the tracking optics. Unlike other projects, we do not forsee the use of laser guide stars (LGS). Nor do we contemplate multi-conjugate adaptive optics (MCAO), at least not initially. Rather, we chose to observe only small regions surrounding suitable natural guide stars (NGS).

There are several reasons for this choice. First, NGS avoids the cone effect (focus anisoplanetism) and its resultant loss of Strehl ratio. Second, even with LGS, a NGS is required to provide wavefront slope correction. Third, the scientific programs to be addressed by LAMA do not require that a guide star be located at arbitrary positions in the sky. Finally, the very high resolution of LAMA means that there will be a large number of resolution elements within the isoplanatic area - more than can be easily sampled with foreseeable infrared arrays.

\subsection{Beam combiner}

The LAMA beam combiner consists of a set of concave mirrors, one per aperture, that receive the afocal beams and cause them to converge to a common focus, as shown in Fig. 3. In order to provide a wide interferometric field of view, the geometrical configuration is Fizeau. This requires that the lateral and longitudinal positions of the exit pupils for each aperture be in the correct locations, forming a configuration that is a homogeneously scaled reproduction of the entrance pupils.

For interferometric telescope systems such as the Very Large Telescope Interferometer (VLTI) and LAMA, the Fizeau requirement is complicated by the fact that the incident rays are not perpendicular to the plane of the array (unless the telescope is observing exactly at the zenith). Therefore, the individual entrance pupils are located at different longitudinal positions with respect to the incident rays, and these positions change with time as the telescope tracks. To preserve the Fizeau geometry, the exit pupil of each beam must be displaced longitudinally by an amount that equals the corresponding displacement of the entrance pupil multiplied by the longitudinal magnification of the telescope, $\alpha^{2}$, where $\alpha$, the transverse magnification, is the ratio of the entrance and exit pupil diameters (the reciprocal of the beam compression factor of the telescope).

The limited pointing range of LAMA simplifies this problem somewhat. For a field angle $\psi$ in the interferometric image, the phase error introduced in a single beam by an error $\Delta s$ in the longitudinal position of the exit pupil is

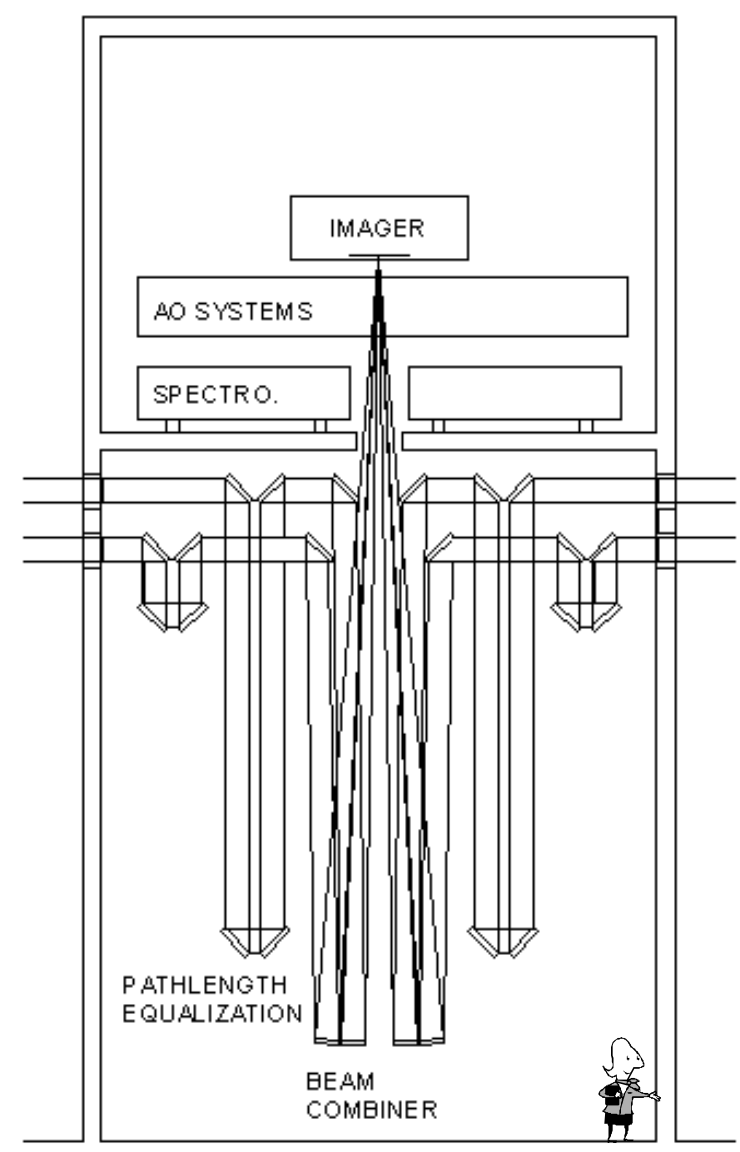

Fig. 3. Possible layout for the beam combiner, path length equalization system, adaptive optic systems, imager and spectrographs.

$$
\Delta \varphi \approx \pi \frac{D}{\lambda} \frac{\Delta s}{s} \psi
$$


where $s$ is the distance from the exit pupil to the focus. From this one can show that, if the variations in longitudinal positions of the entrance pupils are ignored, the RMS phase error in the interferometric image is

$$
\Delta \varphi \approx \frac{\pi \alpha D d}{4 \lambda F} Z \psi
$$

where $F$ is the telescope effective focal length and $Z$ is the zenith angle of the observed field. For the baseline LAMA design, the RMS phase error 30 arcsec from the field center at the maximum zenith angle of 4 degrees is 0.08 which is negligible (less than 1\% reduction in Strehl ratio). However, it is still necessary for the optical system to compensate for the changes in path length, in the compressed beams, introduced by the optical delay lines (described below).

The adaptive optics systems could be simplified considerably if the concave mirrors of the beam combiner are made adaptive. In the baseline design, these mirrors, located at the exit pupils, are $0.4 \mathrm{~m}$ in diameter with a focal length of $13.33 \mathrm{~m}$.

\subsection{Alignment and Phasing}

In order to achieve interferometric imaging, it is necessary that wavefronts in the individual beams converging to the common focus, be correctly aligned and phased. For a conventional MMT, the primary mirrors may be co-phased by a laser metrology system such as that described by Beckers ${ }^{1}$. For the LAMA concept, employing fixed primary mirrors, the optical path length from the focus to different primary mirrors varies by as much as 3 meters (due to the motion of the path length equalization mirrors when tracking an object), rendering such a system impractical. However, even for LAMA, the problem of phasing is in principle straightforward.

Due to the action of the AO systems, the wavefronts in the individual beams converging to the focus are spherical to within a small fraction of a wave. A necessary and sufficient condition to achieve coherence over the entire array is that corresponding wavefronts in the individual beams have the same center and phase to within comparable tolerances. To achieve this, the first four Zernike modes (piston, $\mathrm{x}$ and $\mathrm{y}$ slope, and focus) of each beam must be controlled.

How can this be done, and what tolerances are required? One possible way is to locate a beam splitter in each converging beam to send a portion of the light from the NGS to an image on a CCD camera. At the focal point, the image will be an Airy disk of diameter $\theta \approx \lambda / D$. The position of the centroid of the star image is used to control the tiptilt mirror that steers the beam. It is can be shown that the RMS phase error induced by an angular error $\Delta \theta_{X}$ in the position of the centroid is

$$
\Delta \varphi_{X}=\frac{\pi}{2} \frac{\Delta \theta_{X}}{\theta}
$$

Therefore, to achieve an RMS phase error of less than 0.32, corresponding to a Strehl ratio of $90 \%\left(S \approx \exp \left(-\Delta \varphi^{2}\right)\right)$, it is necessary to control the $x-y$ position to an accuracy of 0.2 times the diameter of the Airy disk. This is easily achievable with a modest signal-to-noise ratio. The required geometrical stability of the cameras is approximately 10 microns which can be achieved by mounting them on a common optical table in a temperature-controlled environment.

Similarly, one can show that the RMS phase error resulting from a focus error that produces a change $\Delta \theta_{F}$ in angular image diameter is

$$
\Delta \varphi_{F}=\frac{\pi}{4 \sqrt{3}} \frac{\Delta \theta_{F}}{\theta} .
$$

This can be measured and controlled by locating the CCD slightly before or after the focus (as in a single-sided wavefront curvature sensor ${ }^{12}$ ).

Finally, one must sense and control the relative phases of the individual beams. This can be done as in a conventional phase-tracking system, by interfering pairs of beams to produce white-light fringes, and using the resulting signals to 
control the piston mode of the adaptive optics systems. Because the phase is determined from the entire 10-meter aperture, the signal-to-noise ratio for the NGS used by the AO systems is more than adequate.

\section{INSTRUMENTS}

The design of instruments for the next generation of very large telescopes is a non-trivial issue. As the telescope size increases, so does the size and cost of the instruments. Fortunately, the distributed-aperture approach of LAMA provides some simplification in this regard.

Two primary instruments are currently envisaged: a multi-band imager and a high resolution spectrograph. The imager will provide simultaneous imaging of a common field of view in at least 6 optical and infrared bands. Located at the combined focus, the imager will use a dichroic mirror to feed separate optical $(0.4-1 \mathrm{um})$ and infrared $(1-2.5 \mathrm{um})$ channels. Within each of these cameras dichroic filters and multiple detector arrays will provide simultaneous imaging in several broad bands. In order to optimize sampling, the pixel size in the infrared channels is twice that of the optical channels. As they cover the same field, the number of optical pixels is four times the number of infrared pixels. Two different image scales are used, corresponding to the two operating modes of LAMA. In coherent mode, the pixel sizes in the optical and infrared channels are 1.0 and 2.0 mas respectively, providing Nyquist sampling at 0.6 and 1.2 um wavelength. In incoherent mode (AO but no interferometry), the image scale is reduced to provide Nyquist sampling of the Airy disks of the individual elements at these same wavelengths. A summary of the proposed parameters of the imager is shown in Table 1.

The high-resolution spectrograph in the baseline LAMA design is intended primarily for visible-wavelength observations of quasar absorption lines. These quasars will be too faint to serve as guide stars for the AO and interferometry, therefore there is no particular advantage to observing the combined image. Therefore, we plan to employ 18 individual cross-dispersed echelle spectrographs each receiving the beam from a single element of the array. This alleviates the problem of designing and building a spectrograph for a 50-meter telescope. The technology of highresolution spectrographs for 10-meter telescopes is already demonstrated.

\section{OPERATION AND PERFORMANCE}

The LAMA telescope will be operated in a rather unique manner. In imaging mode, it will observe fields surrounding stars, of approximately $14^{\text {th }}$ magnitude, that are suitable NGS. Only stars passing within 4 degrees of the zenith will be selected. Table 1 gives the surface density of stars at the galactic pole, the number of stars accessible to LAMA, and the total area of sky within a 15 arcsec radius surrounding these stars. Clearly, there is no shortage of reference stars. Each selected field can be observed for up to $45 \mathrm{~min}$ (depending on the telescope latitude and the declination of the field) in a single night. On other nights, one can observe different fields, or repeat the same fields in order to increase the signal-tonoise ratio. There are many possibilities for survey observations. In "snapshot" mode, one could over the course of a year observe 1800 sq. arcmin (10,800 fields), with 5 min exposures. (assuming $10 \mathrm{hrs}$ per night, 90 dark photometric nights per year and a $24 \times 24$ arcsec field size). Alternatively, one could observe 12 sq. arcmin (48 fields) for a total of 67,500 seconds to create an ultra-deep field.

In spectroscopic mode, one simply points to the target objects as they enter the accessible field of LAMA, follow them until they leave, then acquire the next object. As with imaging, the targets can be required on other nights to build up long integration times. For example, over the course of a year, 100 quasars can be observed for a total integration time of 32,400 seconds

\begin{tabular}{|r|r|r|r|}
\hline magnitude & \multicolumn{1}{|c|}{$\begin{array}{c}\text { density } \\
\left(\mathrm{deg}^{-2}\right)\end{array}$} & $\begin{array}{c}\text { accessible } \\
\text { number }\end{array}$ & $\begin{array}{c}\text { accessible } \\
\text { area }\left(\mathrm{deg}^{2}\right)\end{array}$ \\
\hline 12 & 13 & 31,700 & 1.73 \\
\hline 13 & 27 & 64,800 & 3.53 \\
\hline 14 & 57 & 160,800 & 8.77 \\
\hline 15 & 135 & 324,000 & 17.67 \\
\hline
\end{tabular}

Table 1. Guide star surface densities 
The performance of the baseline LAMA design has been calculated by adopting best estimates for mirror reflectivities, detector quantum efficiencies, adaptive-optics performance with NGS, and by ignoring any loss of Strehl ratio that might arise from errors in alignment and phasing for the interferometric mode. Parameters for the baseline LAMA design are summarized in Table 2. Representative results of the performance calculations appear in Table 3. In this table, $10 \mathrm{~s}$ represents the shortest reasonable exposure time, and 45,000 s the result of observing the same field for 30 minutes each night for 25 nights.

\begin{tabular}{|c|c|c|}
\hline \multicolumn{2}{|l|}{ Array diameter $(d)$} & $54 \mathrm{~m}$ \\
\hline \multicolumn{2}{|l|}{ Element spacing } & $11 \mathrm{~m}$ (hexagonal arrangement) \\
\hline \multicolumn{2}{|l|}{ Filling factor $(f)$} & 0.71 \\
\hline \multicolumn{2}{|l|}{ Effective area } & $1414 \mathrm{~m}^{2}$ (42.4 m equiv. diameter) \\
\hline \multicolumn{2}{|c|}{ Element diameter $(D)$} & $10 \mathrm{~m}$ \\
\hline \multicolumn{2}{|c|}{ Beam combiner } & Fizeau \\
\hline \multicolumn{2}{|c|}{ Pointing range } & Up to 4 degrees from zenith \\
\hline \multicolumn{2}{|c|}{ Unvignetted field of view } & 1 arcmin diameter \\
\hline \multicolumn{2}{|c|}{ Tracking time } & Up to $45 \mathrm{~min}$ \\
\hline \multicolumn{2}{|l|}{ Wavelength range } & $0.5-2.5 \mathrm{um}$ \\
\hline \multirow{2}{*}{$\begin{array}{l}\text { Imager pixel size } \\
\text { (coherent mode) }\end{array}$} & Optical camera & 1.0 mas (0.6 um Nyquist) \\
\hline & IR camera & 2.0 mas (1.2 um Nyquist) \\
\hline \multirow{2}{*}{$\begin{array}{l}\text { Imager pixel size } \\
\text { (incoherent mode) }\end{array}$} & Optical camera & 6.0 mas (0.6 um Nyquist) \\
\hline & IR camera & 12.0 mas ( 0.6 um Nyquist) \\
\hline
\end{tabular}

Table 2. Parameters for the baseline LAMA design

\begin{tabular}{|l|c|c|c|c|c|c|}
\hline Band & \multicolumn{3}{|c|}{ Incoherent mode } & \multicolumn{3}{c|}{ Coherent mode } \\
\hline & $10 \mathrm{~s}$ & $600 \mathrm{~s}$ & $45000 \mathrm{~s}$ & $10 \mathrm{~s}$ & $600 \mathrm{~s}$ & $45000 \mathrm{~s}$ \\
\hline $\mathrm{V}$ & 25.2 & 27.4 & 29.8 & 26.8 & 29.0 & 31.4 \\
\hline $\mathrm{R}$ & 29.1 & 32.3 & 34.6 & 31.6 & 33.9 & 36.2 \\
\hline $\mathrm{I}$ & 30.1 & 32.3 & 34.6 & 31.6 & 33.8 & 36.2 \\
\hline $\mathrm{J}$ & 30.1 & 31.1 & 33.4 & 30.4 & 32.7 & 35.0 \\
\hline $\mathrm{H}$ & 28.1 & 30.3 & 32.7 & 29.7 & 31.9 & 34.2 \\
\hline $\mathrm{K}$ & 27.6 & 29.8 & 32.2 & 29.2 & 31.4 & 33.7 \\
\hline
\end{tabular}

Table 3. Estimated $5 \sigma$ point source-detection limits, in $\mathrm{AB}$ magnitudes $(\mathrm{AB} 31.4=1 \mathrm{nJy})$

\section{SCIENTIFIC POTENTIAL}

There are many compelling scientific reasons to build LAMA. We illustrate this with a brief overview of three examples that demonstrate the scientific potential of this telescope.

\subsection{Very Distant Supernovae}

Supernovae are important to cosmology not only because they are standard candles that can be used to trace the expansion history of the universe but also because the supernova formation rate is directly related to the star formation rate, since supernovae are the explosions of young, massive stars. Thus the incidence of supernovae versus cosmic epoch measures the history of star formation in the universe, which is important because it pinpoints when stars (and the elements they produce) formed in galaxies. Other measures of the star formation history of the universe are highly 
uncertain, because they are subject to various contaminating effects, including effects of cosmological surface brightness dimming and of dust extinction. It is also possible that there exists a class of as yet undiscovered "Population III" supernovae that trace the very first generation of star formation, before nucleosynthesis in the interiors of stars polluted intergalactic space with heavy elements.

Due to both its unique mode of operation and its sensitivity, LAMA is ideally suited to identifying and studying very distant supernovae. By obtaining short exposures of the same fields night after night, LAMA can gradually build up extremely deep images at multiple optical and infrared wavelengths while at the same time providing sensitivity to variable and transient sources like supernovae. Using incoherent beam combination, LAMA can achieve $5 \sigma$ limiting depths for point sources of $A B \approx 31.5$ at optical wavelengths and $A B \approx 29.5$ at near-infrared wavelengths in a single $300 \mathrm{~s}$ exposure. An ensemble of such exposures acquired over the course of a period of months can not only be used to detect but also to classify and measure peak brightnesses of supernovae to very high redshifts. Here the strategy relies on knowing the redshifts of the host galaxies, which are essential for fixing the cosmological time dilation factors of the supernova light curves and for interpreting the observations. But redshifts of the host galaxies can be obtained by exactly the same observations used to identify the supernovae, by applying broad-band photometric-redshift techniques.

For example, an ensemble of $75300 \mathrm{~s}$ exposures spread over a period of five months is in principle sufficient to identify supernovae to redshifts as large as $z \approx 8$. As many as 120 fields covering $30 \mathrm{arcmin}^{2}$ could be monitored in this way (assuming 10 hours per night, $50 \%$ clear nights, and a $30 \times 30 \operatorname{arcsec}^{2}$ field size), yielding up to 288 fields covering 72 $\operatorname{arcmin}^{2}$ per year. Although the incidence of high-redshift supernovae is currently unknown, measures of the rest-frame ultraviolet and $B$-band luminosity densities of high-redshift galaxies in the Hubble Deep Fields suggest that the incidence of supernovae of all redshifts is a least $1 \operatorname{arcmin}^{-2} \mathrm{yr}^{-1}$. (The incidence could be much higher to the extent that the light of galaxies in the Hubble Deep Fields is hidden due to cosmological surface brightness dimming or dust extinction effects.) Thus LAMA could in principle identify up to 72 high-redshift supernovae per year, at redshifts up to $z \approx 8$.

\subsection{The Epoch of Reionization and the Early Intergalactic Medium}

Standard Big Bang cosmology predicts that the universe cooled sufficiently for neutral atoms to form by redshift $z \approx 1000$. Yet sensitive observations of distant quasars indicate that the intergalactic medium is highly ionized at all redshifts $z<5$. Apparently the universe was reionized at some redshift between 5 and 1000, presumably at redshift $z<30$ by ultraviolet light from the first stars and galaxies. Recent observations of quasars of the highest known redshifts suggest that this "epoch of reionization" may have occured at redshifts near $z \approx 6$, but the evidence is so far only tentative. The epoch of reionization is one of the most important events in cosmology - heralding the formation of the first stars, galaxies, and quasars - yet is now essentially unknown and unexplored. The reionization of the universe is of particular significance, because the physical processes involved in galaxy formation probably proceed entirely differently in ionized, as compared with neutral, gas.

Quasars will be of limited utility in studying the intergalactic medium at the epoch of reionization, simply because highredshift quasars are so rare. For example, it is estimated that less than 20 quasars of redshift $z>6$ will be discovered in the entire Sloan Digital Sky Survey of the northern hemisphere sky. Instead, progress toward understanding the intergalactic medium at the epoch of reionization will likely come from studying fainter, lower luminosity objects active galactic nuclei and galaxies - which are much more common than quasars. It is likely that many such objects will be discovered over the coming decade. But the high-resolution spectroscopy needed to apply these objects toward the detection and measurement of the early intergalactic medium is far beyond the capabilities of any existing telescope and will require the light-gathering ability of a large-aperture telescope like LAMA.

LAMA will study the early intergalactic medium in the directions of dozens of very distant galaxies and active galactic nuclei, using the background objects to probe the absorption signatures produced by the intervening medium. Because of its high sensitivity, LAMA will provide the deepest observations of high-redshift background sources ever obtained. It will detect the observational signatures of the reionization of the universe and will determine at what epoch, over what time interval, and by what sources of ultraviolet radiation the universe was reionized. 


\subsection{The Lyman- $\alpha$ Forest}

The "Ly- $\alpha$ forest" absorption systems detected in the spectra of background QSO trace the gaseous content of the universe from the present epoch through to epochs of redshift $z \approx 5$ at which the universe less than approximately $15 \%$ of its current age. Most of the neutral gas content of the universe is contained in the highest column density damped Ly$\alpha$ absorbers, while most of the ionized gas content and perhaps most of the baryons are contained in the lower column density Ly- $\alpha$ absorbers. Observations of quasar absorption lines can provide information about physical conditions of the high-redshift universe that cannot be learned by any other means, including measures of the kinematic state and chemical composition of the interstellar media of high-redshift galaxies. The combination of spectroscopic observations of high-redshift quasar absorption lines with imaging and spectroscopic observations of high-redshift galaxies is a particularly powerful one, because it can reveal the interplay between the gaseous and stellar contents of the universe over most of cosmic time.

Due to its light-gathering ability, LAMA is ideally suited to high-resolution spectroscopy of the Ly- $\alpha$ forest and other quasar absorption line systems. Over the $7 \%$ of the sky accessible to LAMA, there are more than 200 high-redshift $(z>3)$ quasars brighter than $A B(R)=18$. These quasars could provide targets for unprecedented spectroscopy of the Ly- $\alpha$ forest. For example, LAMA could observe a sample of 100 high-redshift quasars for a total exposure time of $69,000 \mathrm{~s}$ each over the course of a year, in lots of $40900 \mathrm{~s}$ exposures per night (assuming 10 hours per night and 50\% clear nights).

With the individual exposures added together, each of the resulting spectra would detect more than 1000 Ly- $\alpha$ absorption lines and dozens of heavy element absorption lines and would surpass the best existing spectra of the Ly- $\alpha$ forest by large factor. These spectra would be used to study a variety of issues, including the chemical content of high-redshift gas and the cosmic chemical evolution of the universe, the abundance of deuterium and the baryon density of the universe, and the formation of the absorption line profiles and the kinematics and dynamics of high-redshift gas. The spectra would also be used to study time variability of the fine structure constant and other physical constants by precisely measuring the wavelength ratios between absorption lines of atomic (or molecular) doublets and multiplets. These observations could confirm or refute recent suggestions that the fine structure constant varies versus epoch.

It could perhaps be even more interesting to examine the observations with the individual exposures not added together, searching for variable or transient events in the Ly- $\alpha$ forest. Such an analysis - which is made possible by the unique mode of operation of LAMA - has never before been performed. There are at least three reasons why Ly- $\alpha$ absorption lines would be expected to vary (in wavelength, line profile shape, or both) with time: (1) because the absorbing gas takes part in the cosmic deceleration (or acceleration) of the universe, (2) because the absorbing gas is subject to peculiar accelerations that are related to the growth of large-scale structure in the universe, and (3) because the absorbing gas drifts transverse to the line of sight. The first of these effects is of particular interest because it in principle provides a way to measure the cosmological parameters (including the equation of state of the universe) that is essentially free of astrophysical systematic effects. The effect is very small, but perhaps within reach of LAMA if systematic measurement effects can be kept under control. The second of these effects is also small, but of magnitude comparable to the first and so also possibly within reach of LAMA.

\section{SUMMARY AND CONCLUSIONS}

This paper has described an alternative approach to the next generation of very large optical telescopes. We propose an array of telescopes with fixed zenith pointing primary mirrors, feeding light to a common focus where it is combined coherently to enable high-resolution imaging over an extended field of view. Employing a system of tracking optics, the telescope acquires and tracks objects passing near the zenith. Over the course time, this telescope can conduct a deep imaging survey of a relatively wide area of sky, and/or an ultra-deep survey of a smaller area to unprecedented detection limits.

This concept has many advantages. Some of the main ones are:

- The required technologies already exist.

- The development risks are low. 
- The cost is low.

- Because the telescope always points near the zenith, atmospheric seeing and extinction are minimized and the effects of atmospheric refraction and dispersion are negligible.

There is only one disadvantage. The telescope accesses a smaller region of the sky than can a fully-steerable instrument. This means that there are some science programs that it cannot do. We believe that this is a relatively minor limitation and that the science programs that the telescope can do are rich, varied, and very exciting.

\section{ACKNOWLEDGEMENTS}

We thank Steve Shectman, John Webb and Jacques Beckers for helpful discussions. PH gratefully acknowledges financial support from the Natural Sciences and Engineering Research Council of Canada.

\section{REFERENCES}

1. J. M. Beckers, "Extremely large multiple mirror telescopes," Proceedings of the Bäckaskog workshop on Extremely Large Telescopes, Eds. Torben Andersen, Arne Ardeberg and Roberto Gilmozzi = ESO Conference and Workshop Proceedings No. 57, pp. 83-90, 1999.

2. E. F. Borra, R. Content, M. J. Drinkwater and S. Szapiel, "A diffraction-limited f/2 1.5 meter diameter liquid mirror," Ap. J. 346, L41-44, 1989.

3. P. Hickson, B. K. Gibson and D. W. Hogg, "Large astronomical liquid mirrors," Pub. Astron. Soc. Pacific. 105, 501-508, 1993.

4. P. Hickson, E. F. Borra, R. Cabanac, R. Content, B. K. Gibson, G. A. H. Walker, "UBC/Laval 2.7 meter liquid mirror telescope,” Ap. J., 436, L201-204

5. P. Hickson, "Wide-field tracking with zenith-pointing telescopes," Mon..Not. Roy. Astron. Soc. 330, 540-546, 2002.

6. F. Roddier and S. T. Ridgeway, "Filling Factor and Signal-to-Noise Ratios in Optical Interferometric Arrays," Pub. Astron. Soc. Pacific. 762, 990-996, 1999.

7. P. Hickson, "Eliminating the Coriolis Effect in Liquid Mirrors," Pub. Astron. Soc. Pacific. 113, 1511-1514, 2001.

8. P. Hickson, E. F. Borra, R. Cabanac, S. C. Chapman, V. de Lapparent, M. K. Mulrooney, G. A. H Walker, "Large Zenith Telescope project: a 6-m mercury-mirror telescope," in Advanced Technology Optical/IR Telescopes VI, Larry M. Stepp; Ed, Proc. SPIE. . 3352, 226-232, 1998.

9. M. K. Mulrooney, "A 3.0 meter liquid-mirror telescope," Ph.D. thesis, Rice University, 2000.

10. E. H. Richardson and C. L. Morbey, "Fifteen degree correcting optics for a 10-meter liquid mirror telescope," Instrumentation for Ground-Based Optical Astronomy, Present and Future. The Ninth Santa Cruz Summer Workshop in Astronomy and Astrophysics, July 13- 24, 1987, Lick Observatory. Ed. L.B. Robinson; SpringerVerlag, New York, NY, p. 720, 1988.

11. E. F. Borra, G. Moretto and M. Wang, "An optical corrector design that allows a fixed telescope to access a large region of the sky," Astron. Astrophys. Suppl. 109, 563-570, 1995.

12. P. Hickson, "Wavefront Sensing from a Single Defocussed Image," Journ. Opt. Soc. Am. A, 11, 1667-1673, 1994. 